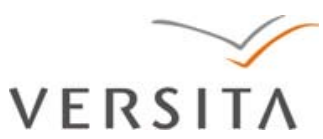

Journal of Official Statistics, Vol. 29, No. 3, 2013, pp. 363-366, DOI: 10.2478/jos-2013-0029

\title{
Discussion
}

\author{
Roderick J. Little ${ }^{1}$
}

I appreciate the opportunity to comment on Mike Brick's review of unit nonresponse adjustments for household surveys. The topic of unit nonresponse in design and analysis has received increased attention with the recent literature on "responsive design", attempting to improve the quality/cost tradeoffs and the deployment of alternative data collection modes to help address the escalating problems of contacting and interviewing respondents. Brick's review assembles a substantial body of research, and provides a useful summary of the "design-based" perspective on unit nonresponse adjustment.

The "critical" part of his "critical review" is mainly directed at our lack of understanding of the processes that lead to nonresponse, and the fact that cognitive research methods are focused on improving questionnaire design, rather than on more general aspects of survey design and analysis. Design approaches to limit missing data are important - I recently chaired a National Research Council panel (National Research Council 2010) where a major focus was design and conduct of clinical trials to reduce the level of missing data. However, is the increase in nonresponse in surveys that much of a mystery? It seems to me clear that people are harder to reach, busier, increasingly inundated with requests to fill out surveys, many from self-serving sources, and just want to be left alone. Characteristics of nonrespondents are important, but as a modeler (Little 2004, 2012), I think the field is too focused on reasons for nonresponse and not enough on modeling the relationship between nonresponse and survey outcomes.

Brick's review embraces the design-based perspective. My "critical review" of the literature would focus more on the limitations of that perspective, both for responsive design and for developing improved nonresponse adjustments. Thus, I liked Brick's quote of Ferber (1949) that "the problem of response bias must be considered with specific reference to a particular question or characteristic", but Brick's review does not really address this key aspect. Models of survey outcomes are largely absent, the emphasis being on modeling the response propensity and associated weighting adjustments. This lack of explicit modeling is characteristic of the design-based perspective - models are implicit and buried in the estimating equations - but attempting to address unit nonresponse without modeling the outcome is for me like trying to tie a shoelace with one hand behind one's back.

I now offer some more specific comments, driven by this overall perspective.

${ }^{1}$ University of Michigan, School of Public Health, Dept of Biostatistics, 1420, Washington Heights, Ann Arbor, MI 48109-2029, USA. Email: rlittle@umich.edu 


\section{Limits of Weighting}

Brick's emphasis on weighting, in the title and the equations he presents, is a reflection of the current state of the field, where unit nonresponse is nearly exclusively handled by weighting adjustments. However, from the modeling perspective, the goal is not to weight, but to predict values of survey variables for nonrespondents, with estimates of uncertainty that reflect imputation error. This philosophy applies whether the nonresponse is at the unit or item level. Some prediction estimators can be expressed using respondent weights, but suitable nonresponse weights often do not have the interpretation of a sampling weight as a sampled case representing a certain number of individuals in the population.

Weighting is limited - it can handle unit nonresponse in a cross-sectional survey, or a monotone pattern such as occurs with attrition in a panel survey, where the variables $Y_{1}, \ldots, Y_{p}$ can be arranged so that $Y_{j}$ is observed for all the cases where $Y_{j+1}$ is observed for $j=1, \ldots, p-1$. It does not handle nonmonotone patterns of missing data well, which is one reason why it is not the approach of choice for item nonresponse. Unit nonresponse in its basic form has a monotone or close to monotone pattern, but nonmonotone patterns can be expected to be more prominent in future, with increased inclusion of information from administrative sources that have their own patterns of missing data. Prediction approaches such as multiple imputation can handle both unit and item nonresponse, and place the emphasis where I believe it belongs, on modeling the survey outcomes. Applying multiple imputation to unit nonresponse is counter to the current orthodoxy, but I note an increasing interest in creating multiple versions of synthetic data sets, where all the data, not just nonrespondent data, are imputed (Rubin 1993; Kinney et al. 2011).

\section{Near-exclusive Focus on Bias Over Variance}

Brick's review mentions precision in a few places, but the emphasis is on bias. He states on page 2 without supporting evidence that "bias is the dominant component of the nonresponse-related error in the estimates". I find this almost exclusive focus on bias odd, particularly since precision is the predominant concern in sample design. A more balanced approach would also consider efficiency, mean squared error, and good confidence interval coverage, but that requires modeling the survey outcomes. The emphasis on bias leads naturally to response propensity weighting and associated $\mathrm{R}$ indicators, but

Table 1. Effect of weighting adjustments on bias and variance of a mean, by strength of association of the adjustment cell variables with response and outcome

\begin{tabular}{lll}
\hline \multirow{2}{*}{ Association with nonresponse } & \multicolumn{2}{c}{ Association with outcome } \\
\cline { 2 - 3 } Low & Low & High \\
& Cell 1 & Cell 3 \\
& Bias: - - & Bias: - - \\
High & Var: - - - & Var: $\downarrow$ \\
& Cell 2 & Cell 4 \\
& Bias: - - - & Bias: $\downarrow$ \\
& Var: $\uparrow$ & Var: $\downarrow$ \\
\hline
\end{tabular}


weighting on a very good predictor of the response propensity that is not related to the outcome is making things worse, leading to inefficient estimates with highly variable weights - see the bottom left cell of Table 1, from Little and Vartivarian (2005) - and confidence intervals with below nominal coverage. On the other hand, other approaches focused on mean squared error, like model-based shrinkage of the weights (e.g., Elliott and Little 2000), or stratifying or weighting based on the predicted mean of an outcome, address both bias and variance (Little 1986; Little and Vartivarian 2005).

Under missing at random (MAR), the response propensity is potentially an important predictor in a model for predicting the outcomes, because misspecification of the relationship between the outcome and the propensity leads to bias - this motivates penalized spline of propensity prediction (An and Little 2004; Zhang and Little 2009, 2011), which models the relationship between the outcome and the propensity as a flexible penalized spline.

\section{Missing Not at Random (MNAR) Models}

Contrary to Brick's discussion in Section 5, I do not think that tinkering with the weights is a fruitful approach to modeling deviations from missing at random. Some estimates under MNAR models can be constructed in a weighted form (an early example is Little 1985), but I think that the best way to address the problem is to explicitly model the joint distribution of the nonresponse indicators and the survey outcomes, as in the proxy pattern-mixture analysis of Andridge and Little (2011). One promising area for improving nonresponse adjustments is the inclusion of proxy and survey process variables, which are often subject to measurement error.

West and Little (2012) address measurement error in auxiliary variables using a patternmixture model. Another simple pattern-mixture approach to modeling deviations from MAR is to apply multiple imputation with offsets to reflect differences in the predictive distribution of outcomes for nonrespondents and respondents. Giusti and Little (2011) describe this approach on a rotating panel survey, with missing income values and a nonmonotonic pattern.

Deviations from MAR will always remain a hard problem, and I agree with Brick that finding good predictors of the outcomes is key. Incidentally, Brick mentions that the approach of Schouten (2007) does not assume MAR, but at a key point in the argument regression coefficients estimated from the respondents are substituted for coefficients defined for the whole sample. This substitution is only justified under the MAR assumption.

Nonresponse adjustments, unit or item, require modeling assumptions. It is a problem of prediction, not weighting, in my opinion.

\section{References}

Elliott, M.R. and Little, R.J.A. (2000). Model-Based Alternatives to Trimming Survey Weights. Journal of Official Statistics, 16, 191-209.

Giusti, C. and Little, R.J. (2011). A Sensitivity Analysis of Nonignorable Nonresponse to Income in a Survey With a Rotating Panel Design. Journal of Official Statistics, 27, $211-229$. 
Kinney, S.K., Reiter, J.P., Reznek, A.P., Miranda, J., Jarmin, R.S., and Abowd, J.M. (2011). Towards Unrestricted Public Use Business Microdata: The Synthetic Longitudinal Business Database. International Statistical Review, 79, 363-384.

Little, R.J. (1985). Nonresponse Adjustments in Longitudinal Surveys: Models for Categorical Data. Bulletin of the International Statistical Institute, Proceedings of the 45th Session: Invited Papers, Section 15.1, 1-18

Little, R.J. (2004). To Model or not to Model? Competing Modes of Inference for Finite Population Sampling. Journal of the American Statistical Association, 99, 546-556.

Little, R.J. (2012). Calibrated Bayes: An Alternative Inferential Paradigm for Official Statistics (with discussion and rejoinder). Journal of Official Statistics, 28, 309-372.

Little, R.J. and An, H. (2004). Robust Likelihood-Based Analysis of Multivariate Data with Missing Values. Statistica Sinica, 14, 949-968.

Little, R.J. and Vartivarian, S. (2005). Does Weighting for Nonresponse Increase the Variance of Survey Means? Survey Methodology, 31, 161-168.

National Research Council (2010). The Prevention and Treatment of Missing Data in Clinical Trials. Panel on Handling Missing Data in Clinical Trials. Washington, D.C. National Academy Press.

Rubin, D.B. (1993). Discussion: Statistical Disclosure Limitation. Journal of Official Statistics, 9, 462-468.

West, B. and Little, R.J. (2012). Nonresponse Adjustment Based on Auxiliary Variables Subject to Error. Applied Statistics, early view. DOI: 10.1111/j.1467-9876. 2012.01058.x

Zhang, G. and Little, R.J. (2009). Extensions of the Penalized Spline of Propensity Prediction Method of Imputation. Biometrics, 65, 911-918, DOI: 10.1111/j.15410420.2008.01155.x.

Zhang, G. and Little, R.J. (2011). A Comparative Study of Doubly-Robust Estimators of the Mean with Missing Data. Journal of Statistical Computation and Simulation, 81, 12, 2039-122058, DOI: 10.1080/00949655.2010.516750. 